\title{
'RB-PALOMA', VARIEDAD DE SORGO BLANCO PARA PRODUCCIÓN DE GRANO Y FORRAJE
}

\author{
'RB PALOMA', A WHITE SORGHUM VARIETY FOR GRAIN AND FORAGE PRODUCTION
}

\author{
Noé Montes-García ${ }^{1 \star}$, Héctor Williams-Alanís ${ }^{2}$, Tomas Moreno-Gallegos ${ }^{3}$, Ma. Eugenia Cisneros-López ${ }^{1}$ y \\ Víctor Pecina-Quintero ${ }^{4}$
}

\begin{abstract}
${ }^{1}$ Campo Experimental Río Bravo, Instituto Nacional de Investigaciones Forestales, Agrícolas y Pecuarias (INIFAP). Km. 61 Carretera Matamoros-Reynosa, Apdo. Postal 172. 88900, Río Bravo, Tamaulipas, México. ${ }^{2}$ Universidad Autónoma de Nuevo León. ${ }^{3}$ Campo Experimental Sur de Sinaloa. ${ }^{4}$ Campo Experimental Bajío, INIFAP. Km. 6.5 Carretera Celaya-San Miguel Allende. Apdo. Postal 112. 38110, Celaya, Guanajuato, México.
\end{abstract}

${ }^{*}$ Autor para correspondencia (montes.noe@inifap.gob.mx)

La sequía tiene un efecto directo en el rendimiento y en la producción de los cultivos agrícolas, y un efecto indirecto en la producción de alimentos, por lo que es necesario disponer de especies vegetales que puedan utilizarse bajo condiciones de escasez de agua y elevadas temperaturas durante el ciclo de cultivo. El sorgo [Sorghum bicolor (L.) Moench] es un cultivo que produce grano y forraje bajo estas condiciones climáticas. En México se siembran anualmente cerca de dos millones de hectáreas de sorgo para grano (SIAP, 2011). De esta superficie, casi $98 \%$ se cultiva con híbridos y el resto con variedades de polinización libre, principalmente para producir forraje.

La principal ventaja de las variedades es que la multiplicación de semilla la pueden hacer los mismos productores, lo que reduce su costo en comparación con el uso de semilla híbrida. Otra ventaja es que el grano de la mayoría de los genotipos introducidos a México por el Instituto Nacional de Investigaciones Forestales, Agrícolas y Pecuarias (INIFAP), provenientes del Instituto Internacional para la Investigación de Cultivos para los Trópicos Semiáridos (International Crops Research Institute for the SemiArid Tropics, ICRISAT) puede utilizarse en la alimentación humana, y que el resto de la planta es un forraje de alta calidad para la engorda de ganado. El INIFAP ha generado y liberado variedades de polinización libre, entre las que destacan 'Blanco 86', 'Pacífico 301', 'Tropical 401', 'Fortuna', 'Costeño 201', 'Perla 101' (Mora et al., 1986; Rodríguez et al., 1988, 1993;
Palacios et al., 2001), y últimamente la variedad 'Gavatero' en Sinaloa (Hernández et al., 2010).

El germoplasma que dio origen a la variedad ' $R B$ Paloma' fue introducido en 1986 al Campo Experimental Valle de Culiacán-INIFAP, en Sinaloa, México, procedente del ICRISAT. Esta variedad fue desarrollada mediante un proceso de selección "genealógica" a partir de un grupo de líneas "segregantes". Dentro de este grupo la parcela 9 mostró buena adaptación a condiciones de riego y de buen temporal o secano, y características agronómicas aceptables como material forrajero, que se identificó como 'Var-9'. Durante el periodo 1995-2001 se desarrollaron ciclos sucesivos de selección genealógica, generalmente dos ciclos por año, con selección durante el ciclo de verano (en condiciones de secano) y el avance generacional (recombinación) del material seleccionado en el ciclo otoño-invierno (OI), hasta lograr una línea homogénea.

Durante el proceso de selección se aplicaron diversos criterios para mejorar la sanidad de planta y el rendimiento de grano y forraje, hasta lograr un material con uniformidad en porte, aspecto de planta y panoja. Su genealogía corresponde a L9-40-42-2-3-1-2-3. La evaluación se efectuó en condiciones de temporal en 2002-2004 en el norte de Tamaulipas, y la validación del rendimiento de 2005 a 2008. En el ciclo OI 2008-2009 se incrementó semilla y en 2010 el SNICS le asignó el registro SOG-191-270510 en el Catálogo Nacional de Variedades Vegetales. 
La descripción fenotípica de la variedad, según los descriptores de la UPOV (Unión de Productores y Obtentores de Variedades), se hizo en el Campo Experimental Río Bravo (255 57' 53” LN, 9801’06” LO, altitud $30 \mathrm{~m}$ ) durante los ciclos OI 2008 y 2009. Desde la emergencia de la plántula hasta la emergencia de la panícula la variedad 'RB Paloma' se caracteriza por carecer de antocianinas en el follaje. Sus hojas son de color verde claro, de textura media, con entrenudos de 8 a $10 \mathrm{~cm}$. En floración las glumas no presentan antocianinas.

Existe pubescencia en la gluma y estigma, y la lema no tiene arista. El color de las anteras y de los estigmas es semejante (verde pálido). El estigma se considera muy corto $(1 \pm 0.010 \mathrm{~mm})$ y de tipo plumoso. Al terminar la floración, el número de flores por panícula es de $1800 \pm$ 200. En madurez, la altura promedio de 'RB-Paloma' es de $170 \pm 19 \mathrm{~cm}$, y su tallo tiene un diámetro de $1.30 \pm 0.30$ $\mathrm{cm}$, sin ramificación ni amacollamiento. La longitud de panoja es de $28.8 \pm 3.2 \mathrm{~cm}$ y presenta moderada tolerancia al acame. El grano es de color blanco-cremoso, sin taninos ni testa; su endospermo es $67 \%$ harinoso y $33 \%$ cristalino; la gluma es larga (cobertura de 69 a $80 \%$ ).

En evaluaciones efectuadas durante el periodo 20052007, el rendimiento de grano promedio de 'RB-Paloma' fue $4711 \mathrm{~kg} \mathrm{ha}^{-1}$ (Cuadro 1). En comparación con otras variedades de grano blanco liberadas por el INIFAP ('Perla 101', 'Costeño 201' y 'Mazatlán 16'), 'RB-Paloma' es superior en longitudes de panoja (19\%) y de excersión (34\%), así como en rendimiento de grano $(31 \%=1112 \mathrm{~kg}$ ha $\left.^{-1}\right)$, además, ha mostrado menor incidencia de enfermedades foliares (25\%). 'RB Paloma' también superó en $10 \%\left(435 \mathrm{~kg} \mathrm{ha}^{-1}\right)$ al promedio de otros testigos comerciales ('Asgrow Ámbar' y 'RB 3030').

Cuadro 1. Promedios de rendimiento de grano y características agronómicas de nueve genotipos de sorgo para grano cultivados en cuatro ambientes bajo temporal en Estación Cuauhtémoc,Tamaulipas.

Características agronómicas

Genotipo Rendimiento de grano $\left(\mathrm{kg} / \mathrm{ha}^{-1}\right)$

(Promedios de cuatro ambientes )

\begin{tabular}{|c|c|c|c|c|c|c|c|c|c|c|c|}
\hline & PV 05 & PV 06 & PV07 & OI 06 & Prom. & $\begin{array}{l}\text { DF } \\
+\end{array}$ & AP & LP & EXC & $\mathrm{CP}$ & ENF \\
\hline 'Ámbar (T)' & 5890 a & $3780 \mathrm{ab}$ & $5253 \mathrm{a}$ & $3835 \mathrm{ab}$ & $4690 \mathrm{a}$ & $67 \mathrm{~b}$ & $151 \mathrm{e}$ & $25.2 \mathrm{ab}$ & $14.1 \mathrm{~d}$ & $2.4 \mathrm{bc}$ & $2.4 \mathrm{~b}$ \\
\hline 'Costeño 201' & $5484 \mathrm{a}$ & $3572 \mathrm{abc}$ & $4283 \mathrm{ab}$ & 3565 bc & $4226 \mathrm{ab}$ & $72 \mathrm{a}$ & $189 \mathrm{c}$ & $21.3 \mathrm{f}$ & $15.3 \mathrm{~cd}$ & $2.4 \mathrm{bc}$ & $2.2 \mathrm{bc}$ \\
\hline 'Mazatlán 16’ & $5831 \mathrm{a}$ & 3208 bc & $4860 \mathrm{a}$ & $2194 \mathrm{e}$ & $4023 \mathrm{ab}$ & $65 \mathrm{~b}$ & $185 \mathrm{~cd}$ & $22.1 \mathrm{def}$ & $17.6 \mathrm{c}$ & $2.5 \mathrm{~b}$ & $2.2 \mathrm{bc}$ \\
\hline 'RB-3030 (T)' & $5565 \mathrm{a}$ & $2747 \mathrm{c}$ & 4030ab & $3107 \mathrm{~cd}$ & $3862 \mathrm{~b}$ & $61 \mathrm{c}$ & $145 \mathrm{ef}$ & $24.7 \mathrm{bc}$ & $12.8 \mathrm{~d}$ & $2.6 \mathrm{~b}$ & $2.5 \mathrm{~b}$ \\
\hline Media & $4810 \mathrm{a}$ & $3314 c$ & $4274 \mathrm{~b}$ & $3124 \mathrm{c}$ & 3880 & 66 & 187 & 23.3 & 18.3 & 2.5 & 2.4 \\
\hline $\mathrm{CV}(\%)$ & 14 & 12 & 13 & 10 & 12 & 2 & 4 & 6 & 13 & 14 & 18 \\
\hline
\end{tabular}

Genotipos con la misma letra en una columna son estadísticamente iguales (Tukey, 0.05). ${ }^{\dagger}$ Promedio de tres ambientes.DF = días a floración; AP $=$ altura de planta $(\mathrm{cm}) ; \mathrm{LP}=$ longitud de panoja $(\mathrm{cm}) ; \mathrm{EXC}=$ excersión $(\mathrm{cm}) ; \mathrm{CP}=$ calificación de planta; ENF =enfermedades foliares. Estas dos últimas características se calificaron en forma visual mediante una escala de 1 al 5 en donde 1 es la mejor y 5 es la más mala; $\mathrm{CV}=$ coeficiente de variación 
En la evaluación de producción de forraje y calidad bromatológica bajo riego efectuada durante el ciclo OI 2010, la producción de materia seca fue $2452 \mathrm{~kg} \mathrm{ha}^{-1}$, superior en $22.6 \%$ a la de los sorgos comerciales. Asimismo, produjo $13.88 \%$ de fibra cruda (superior al híbrido 'Pioneer 83 G19' en $6.6 \%$ ) y $5.2 \%$ de proteína (similar al $6.1 \%$ del testigo). Los niveles de calcio (0.29\%), fósforo $(0.22 \%)$ y cenizas ( $8.9 \%)$, fueron superiores en el 'RB Paloma', en $0.11 \%, 0.04 \%$ y $2.44 \%$, en comparación al testigo. El rendimiento y la calidad bromatológica de forraje ubican a la variedad 'RB Paloma' como un material de doble propósito, cuyo forraje puede ensilarse y así coadyuvaría a la solución del principal problema de la ganadería de la región que es la falta de forraje durante la época seca del año.

La variedad 'RB Paloma' presenta tolerancia a las siguientes enfermedades: ergot o cornezuelo (Claviceps africana), antracnosis (Colletotrichum graminícola) y pudrición carbonosa del tallo (Macrophomina spp.). Lo anterior indica que esta variedad es tolerante a esta compleja gama de enfermedades ocasionadas por hongos, además de presentar mejor adaptación a condiciones ambientales adversas que los híbridos comerciales.

Por otra parte, 'RB Paloma' presenta características propias para consumo humano y elaboración de tortillas, ya que su grano de color blanco (Figura 1) posee un reducido contenido de taninos y polifenoles. El grano de esta variedad presenta un contenido de proteína que varía entre 9.11 y $10.7 \%, 0.30 \%$ de cenizas o minerales, $3.65 \%$ de grasa, $7.31 \%$ de fibra y alrededor de $76 \%$ de carbohidratos. Entre los aminoácidos (g/100 g de proteína) presentes en su grano, predominan: ácido aspártico $(0.77 \mathrm{~g})$, ácido glutámico $(2.20 \mathrm{~g})$, alanina $(0.84$ g), fenilalanina ( $0.50 \mathrm{~g})$, leucina $(1.22 \mathrm{~g})$, cistina $(0.57 \mathrm{~g}) \mathrm{y}$ triptófano $(0.87 \mathrm{~g})$. La digestibilidad del grano es de $87.4 \%$.

La semilla básica y registrada está a disposición de los productores o asociaciones de productores en el Campo Experimental Río Bravo del INIFAP, en Río Bravo, Tamaulipas. Para el desarrollo de 'RB-Paloma' se contó con el apoyo financiero de la Fundación Produce Tamaulipas, A. C. a través del proyecto No. 2016247A intitulado "Obtención de híbridos y variedades de sorgo para grano con tolerancia a sequía y enfermedades para el noreste de México". Tanto en la selección como en las evaluaciones hubo también el apoyo de investigadores del Campo Experimental Valle de Culiacán y del Campo Experimental las Huastecas, ambos del INIFAP.

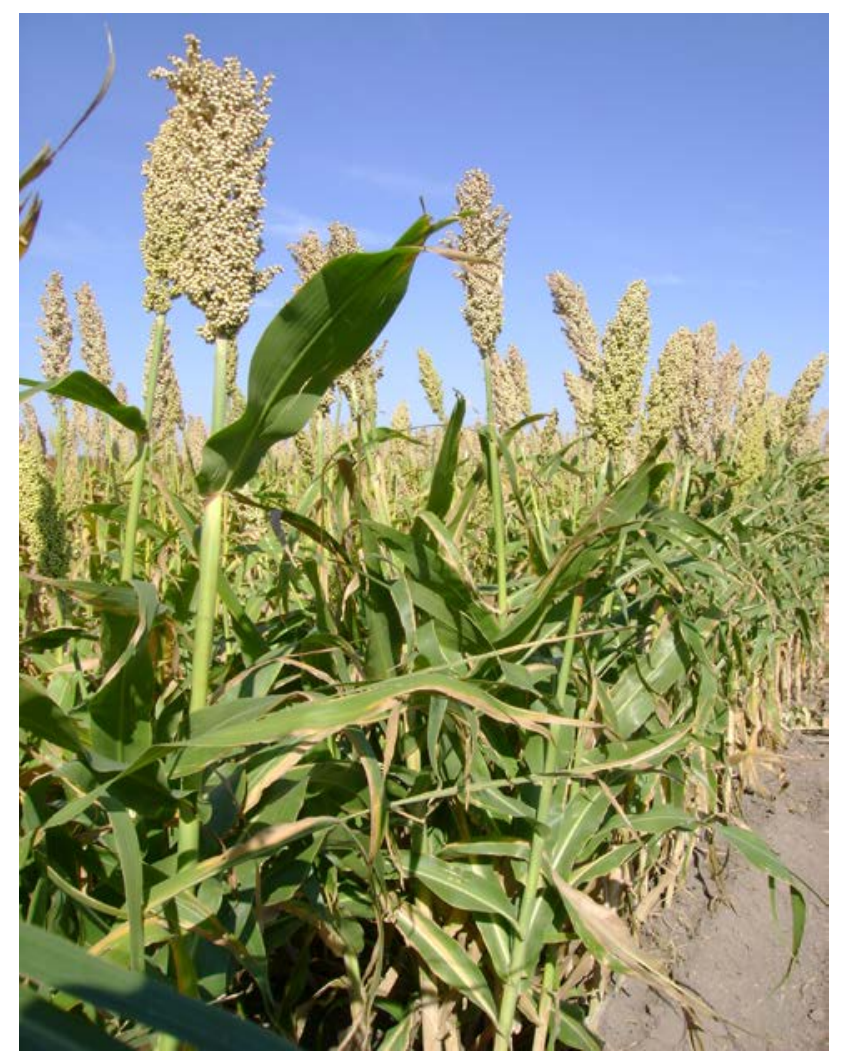

Figura 1. Plantas de la variedad de sorgo de grano blanco 'RB Paloma' crecida en Río Bravo, Tamaulipas.

\section{BIBLIOGRAFÍA}

Hernández E L A, T Moreno G, A Loaiza M, J E Reyes J (2010) Gavatero-203, Nueva variedad de sorgo forrajero para el Estado de Sinaloa. Rev. Mex. Cien. Agríc. 1:727-731.

Mora A R, J Ávila M, V Guiragossian, H Cejudo G (1986) Blanco 86 variedad de sorgo apta para consumo humano. Folleto Técnico No. 6. Campo Experimental Zacatepec. Morelos, México. 12 p.

Palacios V O, T Moreno G, J E Reyes J (2001) Costeño 201, sorgo de doble propósito para temporal en Sinaloa. Campo Experimental Valle de Culiacán, INIFAP. Culiacán, Sinaloa, México. Desplegable para Productores No. 2. 2 p.

Rodríguez H R, J. Trujillo A, P Compton L (1988) Costeño 201, variedad de sorgo apta para consumo humano y adaptada a zonas calidas. Rev. Fitotec. Mex. 11:222-224.

Rodríguez H R, J Trujillo A, P Compton L (1993) Tropical 401: nueva variedad de sorgo para la costa de Jalisco. La Huerta, Jalisco, México. Folleto Técnico Núm. 2. 9 p.

SIAP, Servicio de Información Agroalimentaria y Pesquera (2011) Servicio de Información Agroalimentaria y Pesquera. Información Anual. Sorgo de grano. SAGARPA. Disponible en: www.siap.gob.mx (Marzo 2012). 